\title{
A modifier screen in the Drosophila eye reveals that aPKC interacts with Glued during central synapse formation Lisha Ma ${ }^{\dagger}$, Louise A Johns ${ }^{\dagger}$ and Marcus J Allen*
}

\author{
Address: Cell and Developmental Biology Group, School of Biosciences, University of Kent, Canterbury. UK \\ Email: Lisha Ma - l.ma@kent.ac.uk; Louise A Johns - laj7@kent.ac.uk; Marcus J Allen* - M.J.Allen@kent.ac.uk \\ * Corresponding author †Equal contributors
}

Published: 30 November 2009

BMC Genetics 2009, 10:77 doi:10.1 186/147|-2156-10-77
Received: 6 May 2009

Accepted: 30 November 2009

This article is available from: http://www.biomedcentral.com//47|-2/56/10/77

(c) 2009 Ma et al; licensee BioMed Central Ltd.

This is an Open Access article distributed under the terms of the Creative Commons Attribution License (http://creativecommons.org/licenses/by/2.0), which permits unrestricted use, distribution, and reproduction in any medium, provided the original work is properly cited.

\begin{abstract}
Background: The Glued gene of Drosophila melanogaster encodes the homologue of the vertebrate pI50Glued subunit of dynactin. The Glued' mutation compromises the dynein-dynactin retrograde motor complex and causes disruptions to the adult eye and the CNS, including sensory neurons and the formation of the giant fiber system neural circuit.

Results: We performed a 2-stage genetic screen to identify mutations that modified phenotypes caused by over-expression of a dominant-negative Glued protein. We screened over 34,000 flies and isolated $4 \mathrm{I}$ mutations that enhanced or suppressed an eye phenotype. Of these, 12 were assayed for interactions in the giant fiber system by which they altered a giant fiber morphological phenotype and/or altered synaptic function between the giant fiber and the tergotrochanteral muscle motorneuron. Six showed interactions including a new allele of atypical protein kinase $C$ $(a P K C)$. We show that this cell polarity regulator interacts with Glued during central synapse formation. We have mapped the five other interacting mutations to discrete chromosomal regions.

Conclusion: Our results show that an efficient way to screen for genes involved in central synapse formation is to use a two-step strategy in which a screen for altered eye morphology precedes the analysis of central synaptogenesis. This has highlighted a role for $A P K C$ in the formation of an identified central synapse.
\end{abstract}

\section{Background}

During the development of a neural connection the axon of the growing neuron has to make morphogenic changes to form the presynaptic apparatus needed for efficient synaptic function once it has reached its target cell. This process involves reception of signals by the presynaptic cell followed by precise rearrangements of the cytoskeleton to direct changes in cell shape and control the formation of the presynaptic apparatus (see $[1,2]$ for reviews).

The giant fiber system (GFS) is a unique neural circuit that contains several of the few identified central synapses in
Drosophila and includes the largest in the fly between the giant fiber (GF) interneuron and the leg extensor muscle motorneuron, the tergotrochanteral motorneuron (TTMn), the GF-TTMn synapse [3]. Several studies using over-expression of dominant-negative transgenes, or homozygous adult viable mutations, have recently shed light on signaling mechanisms during the formation of the GF-TTMn synapse. These include the receptors Semaphorin 1a and Roundabout [4,5]; the L-1 type cell-adhesion molecule Neuroglian [6]; the endocytotic and ubiquitin machinery [7-10]; the small GTPase DRac1 [11], and the transcription factor Ken [12]. However, the 
precise mechanisms by which these integrate during synaptogenesis are yet to emerge.

Glued encodes the largest subunit of the retrograde motor dynein-activating complex dynactin $[13,14]$. The Glued $^{1}\left(G l^{1}\right)$ mutation results in a truncated protein product [15] that disrupts the dynein-dynactin complex by binding to dynein and microtubules but fails to bind to cargoes [16]. Mutants have both visual and CNS defects [17-19]. Glued has a key role in formation of the GF presynaptic bend which may involve local cytoskeletal dynamics and rearrangements [20]. Forward genetic screens to identify gene products involved in post-mitotic neural differentiation can be problematic as many of these genes are plieotropic and will have vital functions earlier in development, thus preventing mutants from being recovered. Moreover, many of the genes may well have several functions during differentiation of a single neuronal type. Consequently, mutants, therefore, will exhibit phenotypes that are difficult to interpret. Indeed, this has been shown for the dynein-dynactin complex in the formation of mushroom body neurons [21]. The eye is not required for either viability or fertility of the adult and genetic disruptions targeted to the eye have been exceptionally useful in deducing signaling pathways, for example the sevenless pathway [22], and also in identifying mutant alleles that would cause lethality earlier in development if they were to be expressed throughout the organism [23-25]. This coupled with the fact that it has been estimated that two thirds of the vital genes within the Drosophila melanogaster genome $(\sim 2,500)$ are involved in its development [26], makes the eye invaluable in a primary screen for genes with additional roles in processes such as neural differentiation.

In this study we have undertaken a genetic modifier screen in the adult eye and isolated 12 mutations that either dominantly enhance or suppress a phenotype caused by over-expression of a dominant-negative form of the Glued protein $\left(\mathrm{Gl}^{\mathrm{DN}}\right)$. We assayed these mutations for additional interactions with Glued in the GFS and found that 6 show interactions. Mapping of each modifier mutation is presented. One of the suppressors is an allele of $a P K C$, and we found that other aPKC mutant alleles exhibit suppression of the synaptic phenotype caused by over-expression of GIDN.

\section{Results \\ Screening for modifiers of a truncated Glued (GIDN) over- expression eye phenotype}

$\mathrm{Gl}^{1}$ is a true dominant-negative mutation and the effects of the truncated product produced are dose-sensitive $[17,20,27]$. We previously exploited this by generating a UAS-GlDN transgene to over-express this "poison subunit" using the GAL4-UAS system [28] and showed that disrup- tion of retrograde motor function resulted in synaptic defects in the GFS [20]. Our aim was to identify genes that acted with Glued during GFS formation in the developing CNS. Direct screening for alterations of an adult neural phenotype is problematic and extremely labor-intensive because it would involve an F2 screen. Individual mutant stocks would need to be made and crossed into the appropriate mutant background followed by dissection and staining of the adult CNS.

We therefore reasoned that since $G l^{1}$ affects the development of the eye, monitoring the eye phenotype in a primary F1 screen would provide an excellent read-out for defining interacting loci. Using the eye-specific GMRGAL4 line to target truncated Glued (GlDN) resulted in the generation of adult flies possessing smaller eyes with fused ommatidia and miss-arrayed eye bristles. As expected, this was a more severe phenotype than that seen in $G l^{1}$ mutants (Figure 1C \&1D). This disruption provided a sensitized background in which to base an F1 screen on adult eyes to isolate novel mutations that altered this phenotype. We performed an EMS screen for second-site modifiers that dominantly enhanced or suppressed the over-expression phenotype in the eye (Figure 1A; see materials and methods). We screened over 34,000 flies and obtained both suppressors (Figure 1E) and enhancers (Figure 1F) that reproducibly altered the eye phenotype. We recovered nine lines with homozygous lethal mutations on the second chromosome and seven lines with homozygous lethal mutations on the third chromosome that were either enhancers of Glued (EGs) or suppressors of Glued (SGs). The lethal mutations were most likely the dominant modifiers of the eye phenotype but we could not rule out the posibility of second-site mutations on the chromosomes that were responsible for altering the phenotype. The lines were crossed inter se for complementation. All mutations showed complementation illustrating each of the 16 mutations map to independent loci (data not shown). Twelve of these were analyzed further (see below).

\section{Identification of mutations that interact with $\mathrm{GI}^{l}$ in the adult eye}

Our sensitized screen allowed us to isolate mutations that reproducibly dominantly enhanced or suppressed the eye phenotype caused by over-expression of GlDN. From these lines we wished to identify which mutations dominantly interacted with Glued, rather than simply up- or down-regulated the GAL4/UAS system or interacted with the synthetic GMR element which can cause disruptions if homozygous or if the temperature is raised [29]. To do this we tested whether these mutations could dominantly interact with the $G l^{1}$ allele by making flies heterozygous for both $\mathrm{Gl}^{1}$ and the novel EG or SG mutations and identifying subsequent alterations of the $G l^{1}$ eye phenotype. 


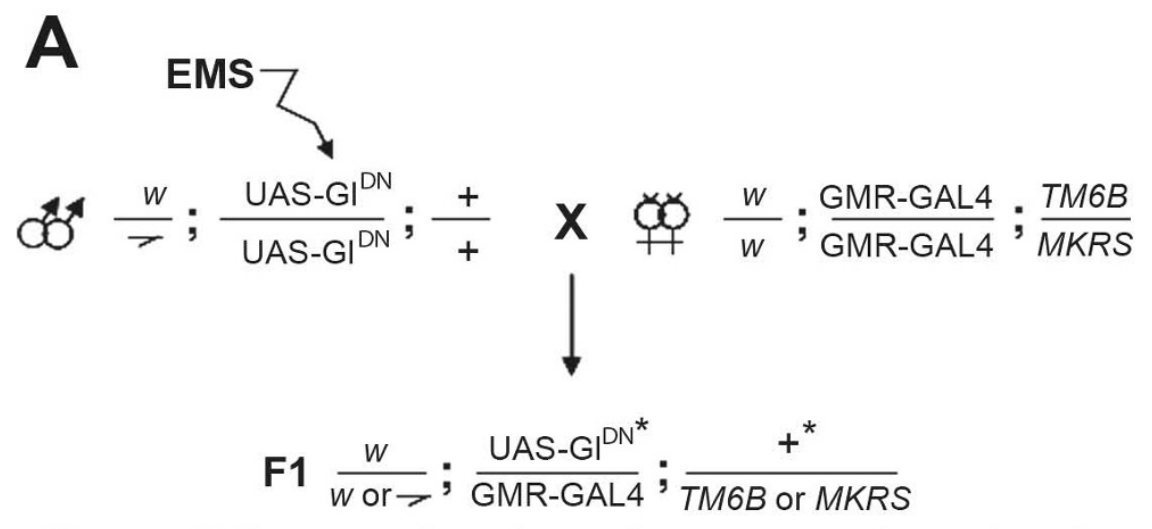

Screened F1 progeny for enhanced or suppressed eye phenotype

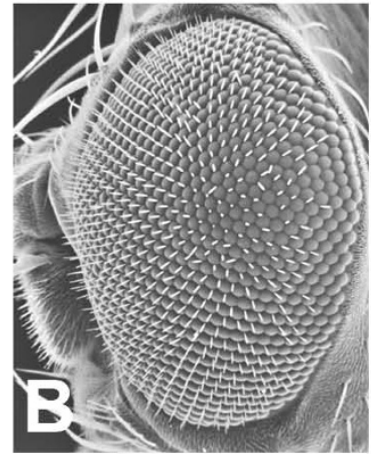

WT

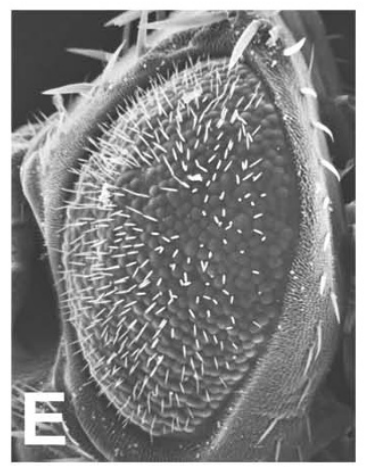

GMR> $\mathrm{GI}^{\mathrm{DN}}$

+Suppressor

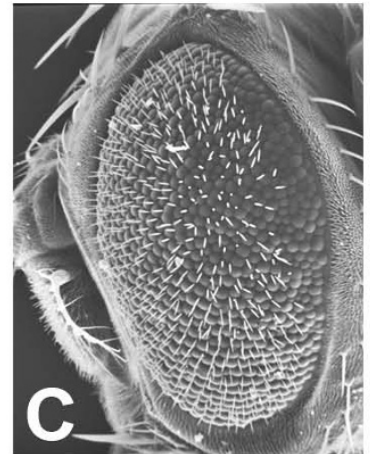

GI'1+

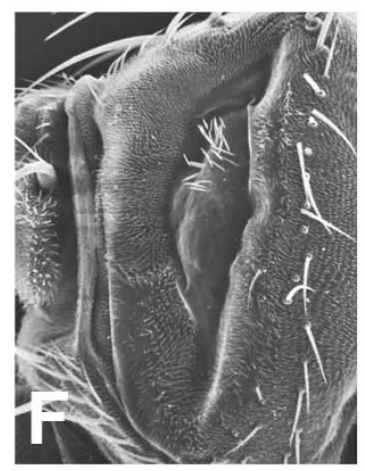

$\mathrm{GMR}>\mathrm{GI}^{\mathrm{DN}}$

+ Enhancer

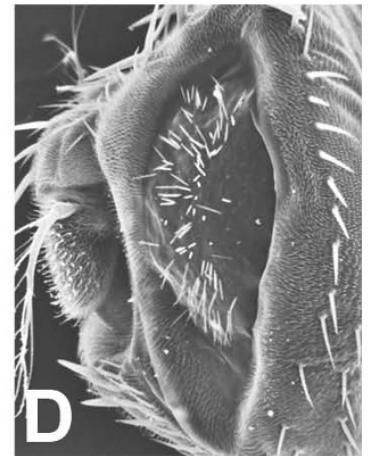

GMR $>\mathrm{GI}^{\mathrm{DN}}$

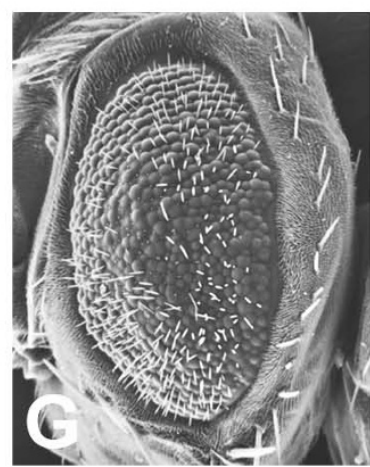

GI'/+

$+\mathrm{EG} 162 /+$

Figure I

The GIDN sensitized screen in the adult eye. (A) Schematic of the screen in which UAS-GIDN males were mutagenized and crossed to GMR-GAL4 virgin females. In the FI generation the flies were scored for any enhancement or suppression of the eye phenotype caused by over-expression of GIDN. (B-D) Scanning electron micrographs of adult eyes. (B) w; UAS-GIDN, showing a wild type eye with a regular array of ommatidia and bristles. (C) Gl//+ exhibiting a roughened, smaller eye. (D) w; GMR-GAL4/UAS-GIDN (abbreviated to GMR >GIDN throughout) showing the much reduced and disorganized eye phenotype used as the basis for the screen. (E) shows the effects of a dominant suppressor (SGI3/+) on the phenotype in (D), and (F) shows the effects of a dominant enhancer (EG37/+). (G) GI/l+; EGI62/+, showing an enhanced phenotype than for GI/+ alone depicted in (C). 
The $G^{1}$ eye phenotype was clearly exacerbated by the enhancer EG162 (Figure 1C \&1G). Other mutations caused more subtle alterations of the $G l^{1}$ adult eye phenotype that were either variable or could not be distinguished when viewed under a dissecting microscope. The eyes of $\mathrm{Gl}^{1} / \mathrm{+}$ individuals show irregular ommatidia and bristle orientation as revealed by SEM (compare Figure 2B to $2 \mathrm{~A}$ ). In addition retinal sections reveal a variable number of rhabdomeres in each ommatidium, which are often reduced in size, as well as clear disruption of the accessory cells. As previously reported, these affects produce a distortion in the overall shape of the ommatidia (Figure 2F), [17]. To determine whether we had isolated interacting mutations we examined whole eyes by SEM and retinal sections by light microscopy from the eyes of $\mathrm{Gl} / 1 /$ flies and those transheterozygous with an enhancer (EG37) and a suppressor (SG13). When EG37 was introduced into the $G l^{1}$ background (EG37/+; Gl1/+) we saw an increase in bristles and an increase in ommatidial fusion and disorganization (Figure 2C). Retinal sections of the eyes from these flies revealed increased disruptions of accessory cells and fused rhabdomeres (Figure 2G). The number of rabdomeres per ommatidium was $4.43 \pm 1.11$

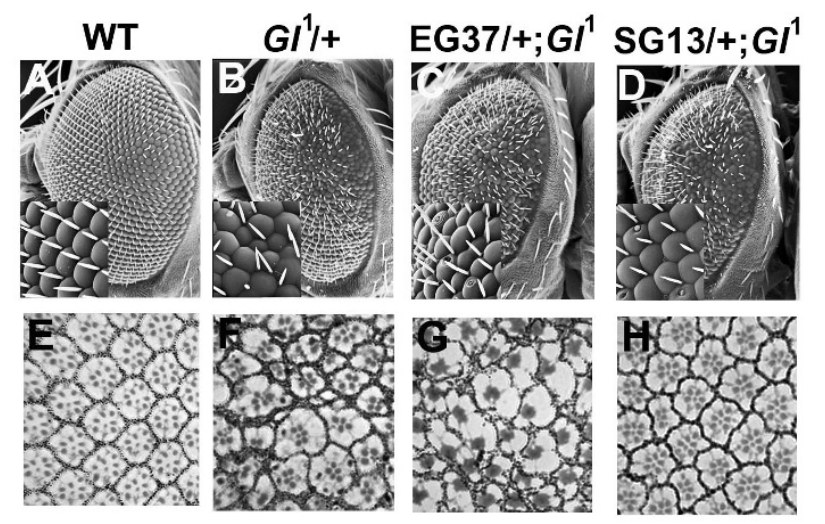

\section{Figure 2}

An enhancer and a suppressor interact with $\mathrm{GI}^{\prime}$ in the adult eye. (A-D) Scanning electron micrographs of adult eyes. (A) Wild type. (B) Gll/+, (C) EG37/+;GI//+, both exhibiting a roughened, smaller eye. (D) $S G I 3 /+; G I /+$ showing a slight amelioration of the GIl phenotype. Insets are higher magnifications. (E-H) Tangential sections of adult eyes. (E) Wild type showing the regular pattern of ommatidial assembly and the stereotyped trapezoidal pattern of the rhabdomeres of the photoreceptor cells (RI-R7). (F) GI//+ eye showing disordered and irregular shaped ommatidia often with aberrant numbers of rhabdomeres. $(G)$ The disorganization is exacerbated in the presence of EG37/+ with the rhabomeres often fused. $(H)$ In the presence of SGI3/+ the ommatidia show a more ordered, regular, pattern with each ommatidia often having the correct array of rhabdomeres (compare with $\mathrm{E}$ and $\mathrm{G}$ ).
( $n=195, \mathrm{P}<0.001)$ compared to those from flies containing $G l^{1} /+$ alone which had 5.03+1.28 $(n=119)$. We also sometimes saw holes between some ommatidia in the sections (data not shown). With SG13 in the $G l^{1}$ background (SG13/+; Gl1/+), the ommatidia and bristles became more ordered than is seen in $G l^{1}$ alone (Figure 2, compare D [inset] with B [inset]) and the sections revealed a return to a more hexagonal lattice of accessory cells and trapezoidal pattern of the rhabdomeres (Figure 2H). These contained an average of $5.89 \pm 1.13(\mathrm{n}=195, \mathrm{P}<0.001)$ rhabdomeres per ommatidium. These data indicated that, for at least two of our mutations, we had isolated loci that genetically interact with Glued during eye development. A third suppressor, SG46, was also analysed in this way and also showed supression of the $\mathrm{Gl}^{1}$ eye phenotype (data not shown).

\section{Mutations that interact with Glued in the Giant Fiber System and alter axon morphology}

The presynaptic terminal of the GF-TTMn synapse is a distinctive bend at the end of the GF axon closely apposed to the TTMn dendrite [30]. Several studies have reported that this bend is often absent or altered when the two neurons fail to form a proper synapse $[4,6,7,9,11,20,31]$. Since our main aim was to identify genes involved in synapse formation within the GFS we next performed morphological analysis of the giant fiber neurons in adult flies carrying an EG or SG mutation and with disrupted Glued function.

We used the GF-specific GAL4 enhancer-trap line, A307 [GAL4] (hereafter referred to as A307), to identify alterations of the GF morphological phenotype, brought about by $\mathrm{Gl}^{\mathrm{DN}}$ over-expression, in the presence of the dominant enhancers or suppressors (Figure 3, Table 1). Our previous work indicated that there are two aspects to the phenotype observed when GIDN is targeted to the GF. First, the distinctive terminal bend does not form after 48 hrs of pupal development indicating that synaptogenesis with the TTMn is defective. Second, as the GF axon develops during later pupal stages, the tip swells, often to several times the axon diameter, presumably because of a buildup of cellular material that the motor is unable to move toward the cell body [20]. Like many GAL4-generated phenotypes there is some variation between preparations, most notably on the extent of axon swelling (compare Figure $3 \mathrm{C}$ and $6 \mathrm{~B}$ ). While both the lack of terminal bend and axon swelling are a result of retrograde motor disruption, any link between the two phenotypes is unclear. Both SG13/+ and SG46/+ showed suppression of these two phenotypes when GIDN was expressed using A307 (Figure $3 \mathrm{~F} \& 3 \mathrm{H}$, Table 1). Swollen axon tips were rarely seen and the axons were either "bendless, " or showed at least one bend in a preparation (Figure 3F [arrowhead] \&3H, Table $1)$, a phenotype not seen when the dominant-negative subunit is expressed alone (Table 1). SG58/+ showed a 

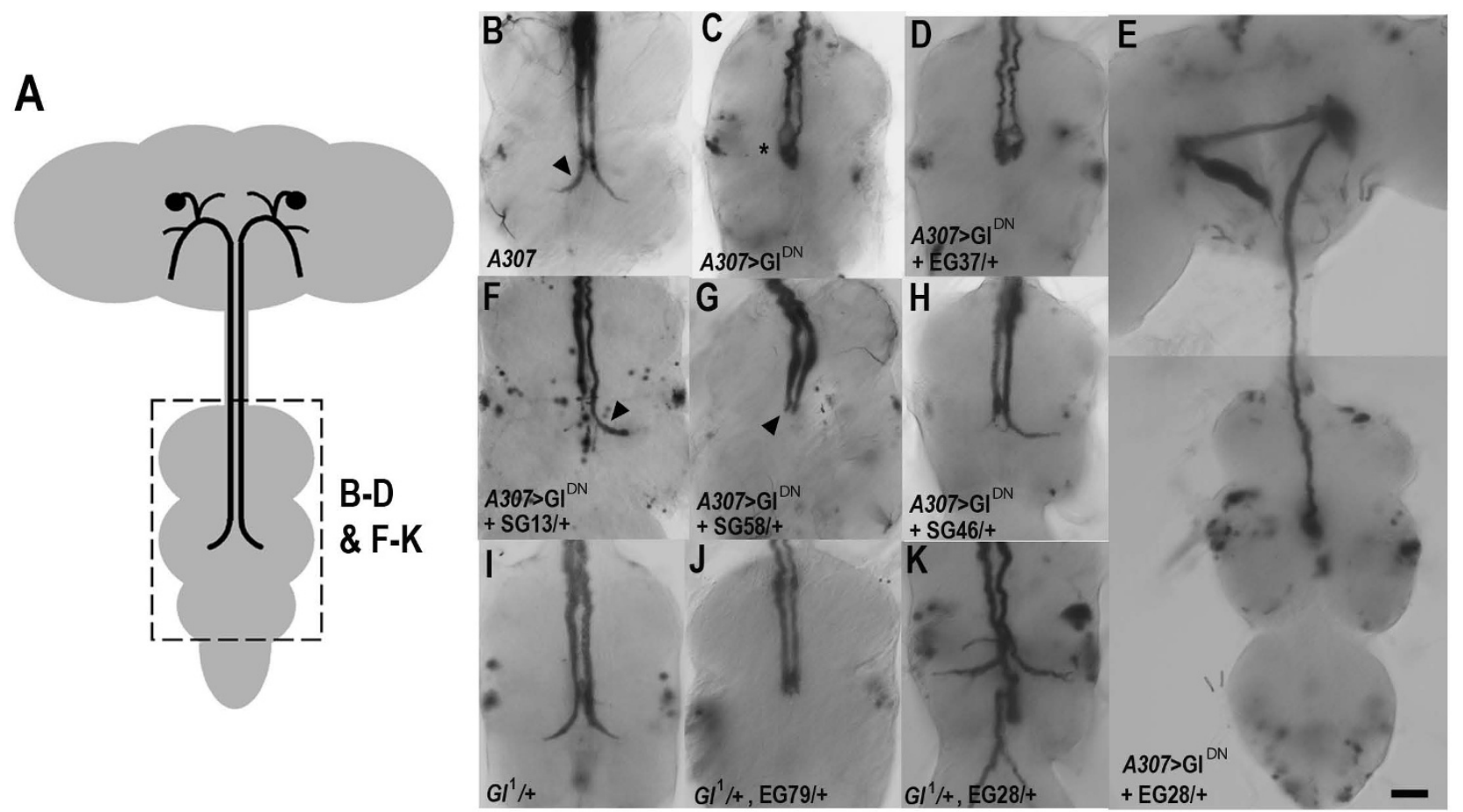

Figure 3

Mutations that interact with Glued and alter GF axon morphology. (A) Schematic of the adult CNS with the GFs indicated. Hatched box indicates the approximate area of the ventral ganglia depicted in B-D \& F-K. (B-K) Dissected adult nervous systems stained for LacZ expression. (B) UAS-LacZ; A307 control showing normal GFs with their characteristic bends in the mesothoracic neuromere (arrowhead) where the GF synapses with the TTMn. (C) A fly also expressing UAS-GIDN exhibits swollen, bendless, axon tips (asterisk). (D) The introduction of EG37/+ did not noticeably enhance the swollen axon phenotype. (E) The whole nervous system is shown for this preparation in which the introduction of EG28/+ results in a more severe phenotype with one GF remaining in the brain. (F-H) Three different suppressors showing amelioration of the swollen axon phenotype. Note that GFs from specimens carrying SG I3/+ or SG46/+ show normal diameter axons and sometimes a terminal bend (arrowhead) and that the GFs from specimens carrying SG58/+ also exhibit normal diameter axons. (I) A fly carrying the GI' mutation exhibits wild type GF morphology. (J\&K) preparations from flies carrying GI' and an enhancer sometimes showed enhancement or altered GF phenotypes (see Table I and text for details). Scale bar is $5 \mu \mathrm{m}$.

mild suppression of the GF morphology phenotype with consistently fewer neurons exhibiting swollen axons (Table 1, see below). However no bends or partial bends were observed (Figure 3G). We could not unequivocally rule out the possibility of these mutations altering the effectiveness of the GAL4/UAS system, however, the phenotypes observed for SG13/+ and SG46/+ were different from those seen if GAL4 expression was suppressed by reducing temperature (M.J.A unpublished observations). SG16/+ showed no rescue of the GF morphology phenotype, suggesting that this mutation does not interact with Glued in the GFS. The EG162/+ and EG165/+ caused dominant lethality in the A307>GIDN background (Table 1) presumably because they affected other cells expressing GIDN in the A307 line that eliminated viability. 20\% (4/ 20) of EG28/+ preparations exhibited GF axons which remained in the brain (Figure 3E). This is likely to be due to a failure to exit the brain on outgrowth, or retraction after a failure in synatogenesis. No obvious enhancement of the GF phenotype was observed with EG37/+ (Figure 3D).

We reasoned that mutations in genes involved in synaptogenesis may not greatly enhance the already severe GlDN synaptic phenotype. Mutations in genes that have a role earlier in development, for example in axon guidance, may give rise to a discernable enhancement, such as incorrect GF axon growth as was the case for EG28/+. In Gl mutants the GFs look morphologically normal, although they have GF-TTMn synaptic defects [20]. Therefore, we tested for dominant effects of the EG mutations on the morphology of the GFs in a $\mathrm{Gl}^{1} /+$ background by generat- 
Table I: Effects of enhancers and suppressors on GFS morphology

\begin{tabular}{lcccc}
\hline & \multicolumn{4}{c}{ GF morphology in A307>GIDN background } \\
\hline Enhancer or suppressor & n† & Swollen "bendless" axons (\%) & "Bendless" axons (\%) & Partial or "Wild type" bends (\%) \\
\hline None & 29 & 86 & 14 & 0 \\
EG28/+ & 38 & $97 *$ & 3 & 0 \\
EG37/+ & 24 & 92 & 8 & 0 \\
EGI62/+ & - & Lethal & Lethal & Lethal \\
EGI65/+ & - & Lethal & 17 & Lethal \\
SGI3/+ & 42 & 88 & 12 & 03 \\
SGI6/+ & 32 & 23 & 32 & 45 \\
SG46/+ & 40 & 65 & 35 & 0 \\
SG58/+ & 43 & & \\
\hline
\end{tabular}

GF morphology in GI/1+ background

\begin{tabular}{llccc}
\hline 3rd C'some enhancer & nf & Swollen "bendless" axons (\%) & "Bendless" axons (\%) & Partial or "Wild type" bends (\%) \\
\hline None & 36 & 0 & 8 & 92 \\
EG7/+ & 24 & 0 & 25 & 75 \\
EG28/+ & 32 & 0 & 3 & 97 \\
EG56/+ & 23 & 0 & 13 & 87 \\
EG79/+ & 22 & 0 & 18 & 82 \\
\hline
\end{tabular}

†GFs were scored individually since the two neurons within one CNS could have different phenotypes.

* some axons failed to exit the brain (see figure $3 \mathrm{~F}$ ).

ing flies with A307, UAS-LacZ, Gl1 and our third chromosome EGs (Figure 3I-K, Table 1). We saw an increase in the frequency of "bendless" axons in 3 of 4 EG mutations tested, suggesting an enhancement of the $\mathrm{Gl}^{1} /+$ phenotype, but no appearance of the swellings seen with overexpression of the dominant-negative subunit. Unusually, preparations containing EG28/+ and $G l^{1} /+$ exhibited a reduction in "bendless" axons and often had ectopic axonal branching (Figure $3 \mathrm{~K}$ ). Taken together with the phenotype seen with GlDN (see above), we presume this mutation is at a locus that has a role in axon growth and/ or guidance. We were unable to do this with the mutations on chromosome two, using any GAL4 GF marker lines, since the mutations are on the same chromosome as the UAS-GIDN insert resulting in over-expression of GIDN in any cell that express GAL4 and contain the mutation. The UAS-GIDN element and the interacting mutations would need to be separated onto different chromosomes by either recombination or precise excision of the P-element containing the UAS-GIDN transgene. As an alternative, morphology of the GF can be observed using dyefilling techniques [31-33]. However, these techniques are labor-intensive and were not practicable for screening purposes.

\section{Mutations that interact with Glued in the Giant Fiber System and alter synaptic function}

We used electrophysiology to assay GFS function, which, in combination with morphological analysis, can reveal abnormalities of synaptogenesis during development [4$7,9,11,12,20]$. A severe GF-TTMn synaptic phenotype is caused by over-expression of GlDN; some flies fail to respond to brain stimulation and those that do respond exhibit a long response latency and show only a single response or poor following to repetitive stimuli ([20]; Figure 4). We put the SG mutations which had exhibited morphological rescue into the A307/UAS-GIDN background. Corresponding with the observed morphological suppression (Figure 3F \&3H, Table 1), flies also containing either SG13/+ or SG46/+ exhibited an increase in GFTTMn synaptic function. All preparations responded to brain stimulation and had shorter response latencies and increased following at 100 and $250 \mathrm{~Hz}$ than is seen with simple over-expression of GlDN (Figure 4). SG58/+ also exhibited a detectable increase in synaptic function with all preparations responding upon stimulation, a slight reduction in the long latency seen when $\mathrm{Gl}^{\mathrm{DN}}$ is overexpressed and also a corresponding increase in following to repetitive stimuli which was significant at $100 \mathrm{~Hz}$ (Figure 4).

Gl $l^{1} /+$ mutants show a functional defect in the GF-TTMn synapse when tested using electrophysiology. They exhibit a response latency not significantly different from wild type ( $\chi=1.1 \mathrm{~ms}, n=8)$ to brain stimulation, but do not follow 1:1 on stimulation at a frequency of $250 \mathrm{~Hz}$ as observed in wild type flies ([20]; Figure 5A \&5C). We crossed the EGs into the $\mathrm{Gl}^{1} /+$ background to observe any 
A

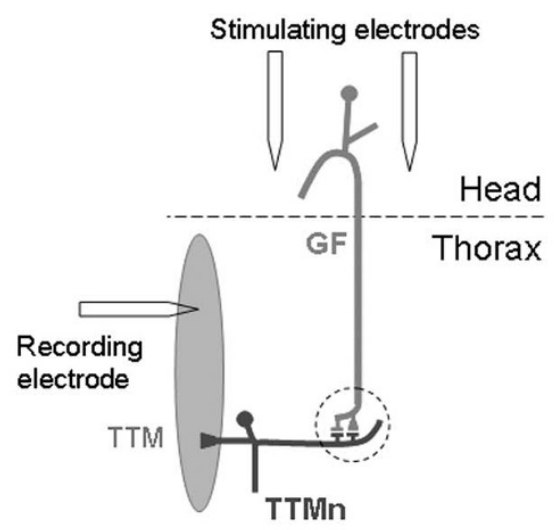

B

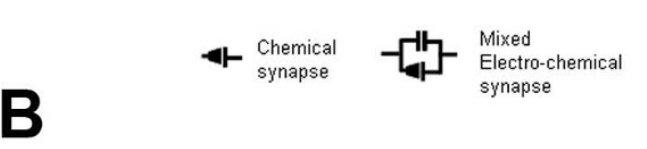

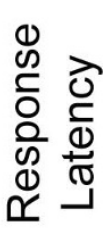

$250 \mathrm{~Hz}$
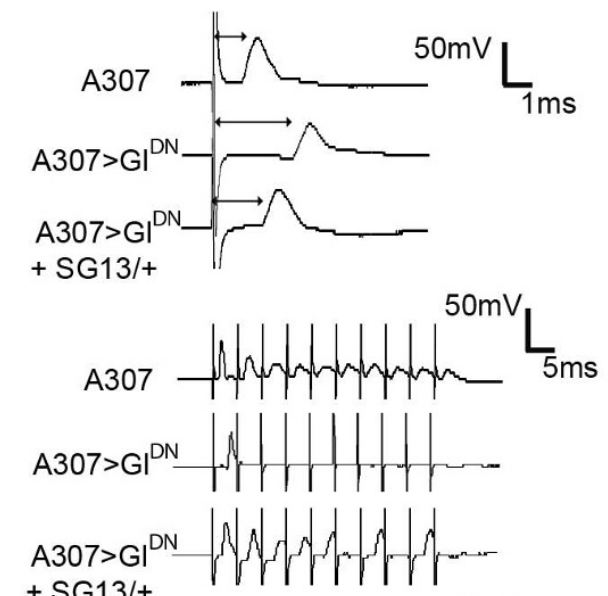

$+\mathrm{SG} 13 /+$

$100 \mathrm{~Hz}$

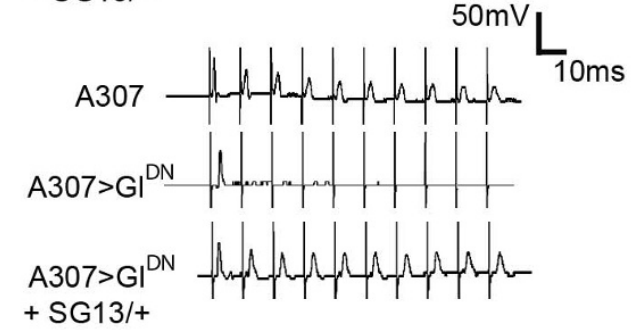

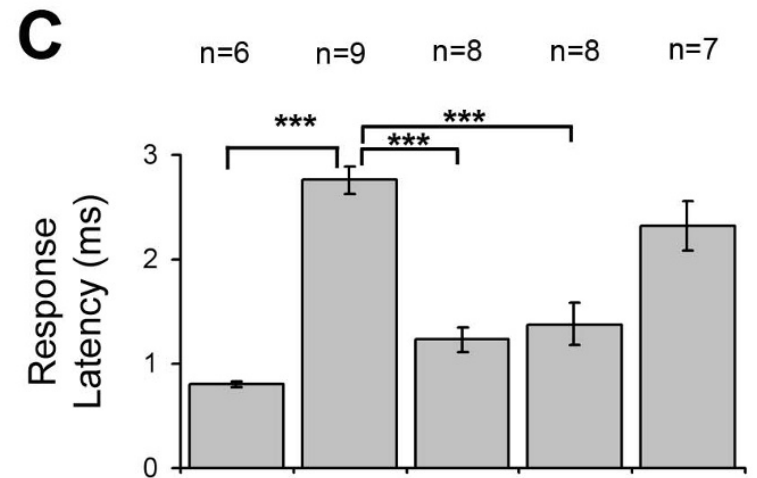
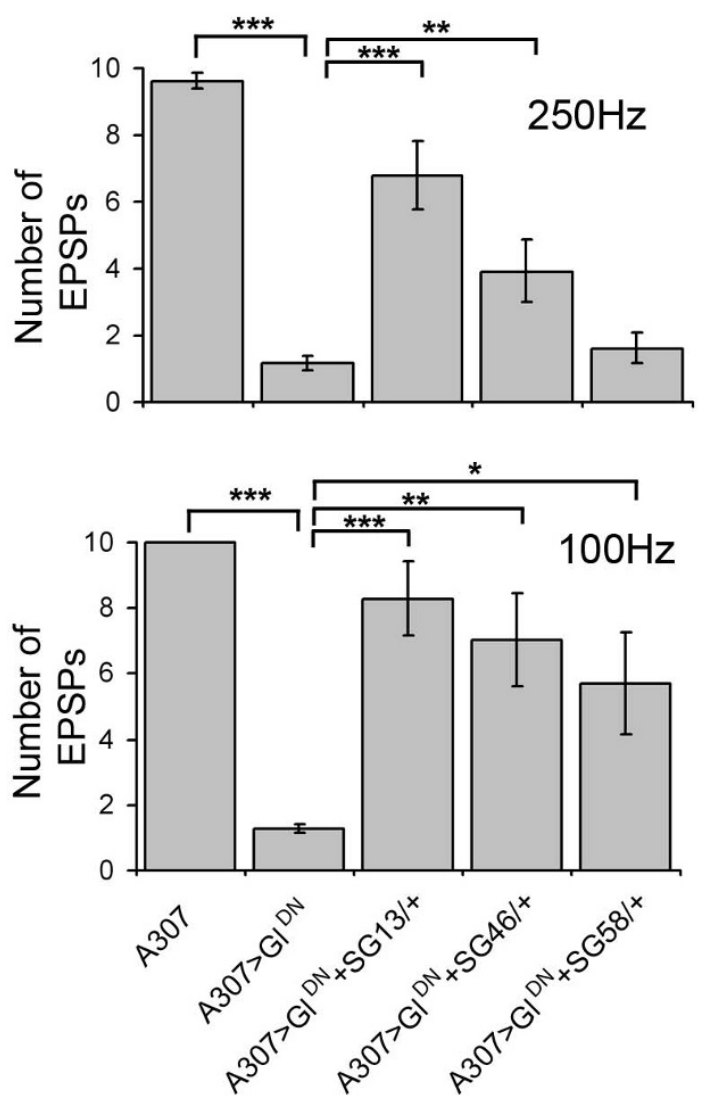

\section{Figure 4}

Mutations that suppress the electrophysiological phenotype seen in A307> GIDN adult flies. (A) Schematic depicting the GF, TTMn and the positioning of the stimulating and recording electrodes to test the function of the GF-TTMn synapse (circled). (B) Traces from individual flies showing the response latency upon a single stimulus and following to 10 stimuli at 250 and $100 \mathrm{~Hz}$. A fly containing $A 307$ alone shows a short response latency and I:I following at 250 and $100 \mathrm{~Hz}$. A fly expressing GIDN exhibits a longer latency and gives only a single response or follows poorly at either 100 or $250 \mathrm{~Hz}$. The addition of SG I 3 / + reduces the response latency back toward that of the control fly and increases following to stimuli at both 250 and $100 \mathrm{~Hz}$. (C) Histograms showing the average response latencies and following to 10 stimuli at the two frequencies for the three suppressors tested. As indicated, responses from A307> GIDN flies were compared to A307-containing flies and all others were compared to $A 307>$ GIDN flies. $* P<0.05$, **P $<0.0$ I, $* * * P<0.001$ in unpaired Student's t-test. 
enhancement of this synaptic phenotype. Because this test did not rely on GAL4 expression we were able to assay any of the EG mutations, regardless of their chromosomal location. All of the EGs tested showed significantly increased reponse latencies (Figure 5B) and exhibited poor following. To analyse this more carefully we looked at the probability of a response being seen with each sequential stimulus at $250 \mathrm{~Hz}$. When 10 stimuli were given to $\mathrm{Gl}^{1} /+$ mutants, the flies demonstrated a depression in response to stimuli 1 through 10 with a plateau after stimulus 6 (Figure 5D), probably due to TTMn not always reaching threshold. This resulted in a 30\% (probability 0.3 ) chance of responding to the last 4 stimuli for $\mathrm{Gl}^{1 /} /+$ flies whereas wild type flies will typically have > $90 \%$ (probability $>0.9$ ) chance of responding (Figure 5D). When EG37/+ or EG162/+ were trans-heterozygous with $\mathrm{Gl}^{1 /} /+$ an enhancement of the phenotype was seen since $100 \%$ of $\mathrm{Gl}^{1} /+$ flies responded to stimulus number 3 whereas the probability of responding fell to 0.6 with EG37/+ and $<0.2$ with EG162/+ added (Figure 5D). We used root mean square deviation (RMSD) to measure the differences in the depression curves and compared them to that seen with $\mathrm{Gl}^{1} /+$. The average deviation between $\mathrm{Gl}^{1 /+}$; EG37/+ and $\mathrm{Gl}^{1 /+}$ was $0.23(\mathrm{P}<0.1)$ and between $\mathrm{Gl}^{1 /+}$; EG162/+ and $\mathrm{Gl}^{1} /+$ was $0.48(\mathrm{P}<0.001)$. For most of the other enhancers tested there was no significant enhancement of the depression seen with $\mathrm{Gl}^{1} /+$ alone (Figure 5E). This included EG28/+ which had given outgrowth/retraction phenotypes when combined with $\mathrm{Gl}^{\mathrm{DN}}$ (see Figure 3). Two of the EGs, EG56/+ \& EG79/+, seemed to suppress the depression (Figure $5 \mathrm{~F}$ ) despite the fact that they caused an increase in the reponse latency (Figure 5B). The reason for this is not known.

\section{aPKC, but not $\mathrm{Su}(\mathrm{H})$, interacts with Glued in synapse formation}

We mapped the six mutations that showed significant interactions with Glued in the GFS using deficiencies and known lethal alleles (see materials and methods). This was assuming that the lethality was associated with the enhancer or supressor. A caveat being that lethality could be due to a second mutation and the interaction with Glued due to a viable allele. Two of the mutations mapped to known genes, $a P K C$ and $S u(H)$, and the others were mapped to small regions on chromosome two or three (Table 2). To determine whether the suppression of the GIDN phenotype in the GF by SG58 was due to the mutation in $a P K C$ we recombined both the $a P K C^{06403}$ and $a P K C^{\mathrm{EY} 22496}$ alleles onto the UAS-GIDN chromosome and crossed these lines to A307. Morphological examination of the GF axons revealed that they were less swollen than seen in A307>UAS-GIDN preparations and occasionally were seen to have a bend (Figure 6). GF-TTMn synaptic function was also increased compared to A307>G ${ }^{\mathrm{DN}}$ flies. When tested using electrophysiology, 89\% (16/18) of
A307> GlDN, aPKC $06403 /+$ flies responded upon GF stimulation compared to $66 \%(10 / 15)$ of $A 307>$ GlDN flies and they exhibited a slightly reduced latency (Figure 6F \&6G) and a statistically significant increase in following at 100 $\mathrm{Hz}$ (Figure 6F \&6H). With the aPKC $\mathrm{EY}^{22496}$ allele, weaker suppression was seen with $78 \%(14 / 18)$ responding and they exhibited an increase in following at $100 \mathrm{~Hz}$ (Figure $6 \mathrm{~F} \& 6 \mathrm{H})$. Overall, the results show weak support for the $a P K C$ interaction with A307>UAS-GIDN from the $a P K C^{06403}$ allele and weaker support from the $a P K C^{\mathrm{EY} 22496}$ allele, however, they are very similar to the effect of the SG58/+ (Figures $3 \& 4$ ) and indicate that $a P K C$ interacts with Glued genetically to alleviate the phenotype caused by the over-expression of the poison subunit.

To determine whether the enhancement of the $G l^{1} /+$ phenotype in the GF by EG37/+ was due to the mutation in $\mathrm{Su}(\mathrm{H})$ we generated $\mathrm{Su}(\mathrm{H})^{1 /} /+; G l^{1} /+$ double heterozygotes and again looked at both the morphology of the GFs, with our A307 line, and performed electrophysiology to assay the function of the GF-TTMn synapse. No enhancement of the $\mathrm{Gl}^{1} / \mathrm{+}$ phenotype was observed in these flies (data not shown). We cannot therefore rule out the presence of a second unmapped mutation on the EG37 chromosome. Alternatively, the mutation in EG37 may have different properties than the $S u(H)^{1}$ allele.

\section{Discussion}

The success of our two-stage screening approach may have been facilitated by the fact that Glued has a plethora of distinct roles during eye development, including organizing optic neural architecture $[17,34,35]$ and an involvement in the formation of sensory neuronal circuits $[18,19]$. Therefore we had an eye phenotype on which to base the screen. However, this does not preclude such a method being used for identifying genes involved in other aspects of neural differentiation. We found that $50 \%$ (6/12) of the isolated mutation-containing chromosomes that altered the eye phenotype also altered GFS phenotypes when tested.

The over-expression of the truncated Glued protein caused strong phenotypes in both the eye and GF neurons, greater than those caused by heterozygosity for the dominant $G l^{1}$ allele. This is likely to be due to the GAL4UAS system producing many more molecules of the truncated product than $G l^{1} /+$ cells in which, theoretically, a maximum of half of the Glued molecules will be truncated. Consistent with this observation, both the suppressors and enhancers isolated during this screen showed stronger effects on GlDN eye phenotypes than on those produced by $\mathrm{Gl}^{1}$. Determining interactions with the Gl11allele also allowed us to confirm GAL4-independent interactions with the Glued locus. For all of the mutations (with the exception of EG162), the alterations of the 

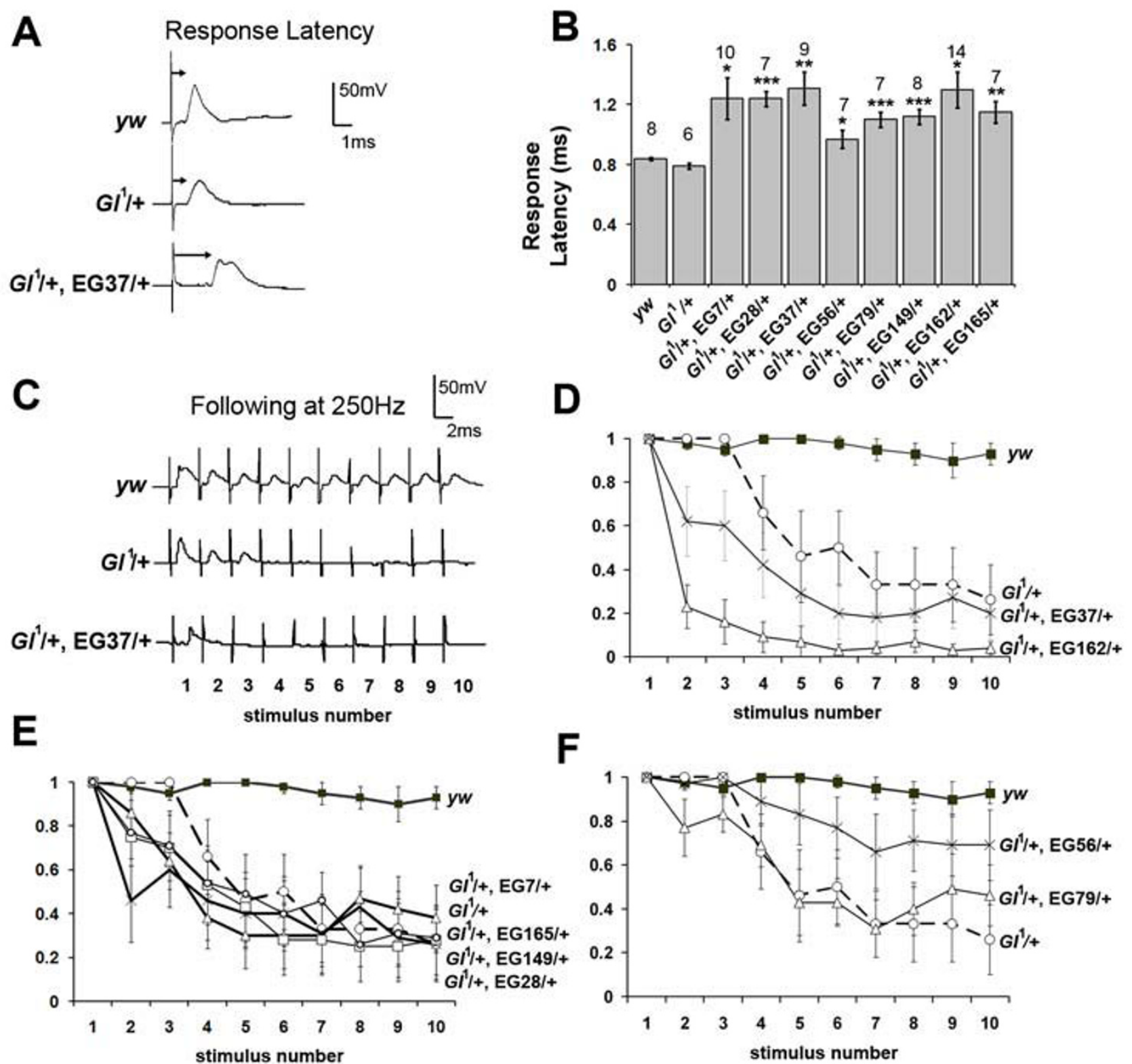

Figure 5

Mutations that enhance the electrophysiological phenotype seen in GI/+ adult flies. (A) Traces from individual flies showing the response latency upon a single stimulus. Both yw controls and $G I^{1 /} /+$ flies show short response latencies. The addition of EG37/+ into the Gll background causes an increase in the latency. (B) Histograms showing the average response latencies recorded for all enhancers in the Gll background. The number of preparations tested $(n)$ is given above each bar and latencies for flies containing $\mathrm{Gl} / \mathrm{l}$ plus enhancers compared to flies containing $\mathrm{Gl} / \mathrm{+}$ alone. *P $<0.05$, **P $<0.0 \mathrm{I}$, ***P $<0.00 \mathrm{I}$ in unpaired Student's t-test. (C) Traces from individual flies showing following to 10 stimuli at $250 \mathrm{~Hz}$. yw controls usually gave a response to each stimulus $(I-I 0)$ whereas this was depressed in $G / 1 /+$ individuals which often gave a response to stimuli I-3 and then failed to respond to the remaining 7 stimuli. The addition of EG37/+ enhances this effect. (D-F) Graphs showing the average probability of a response for stimuli I through 10 at $250 \mathrm{~Hz}$. The data for $y w$ and Gl//+ flies is shown in each panel for comparison against flies trans-heterozygous for $\mathrm{Gl} / \mathrm{l}+$ and the various enhancers. Enhancers EG37/+ and EG I62/+ seemed to increase the depression seen in $\mathrm{Gl}^{\mathrm{l}} /+$ flies with a decrease in the probability of obtaining a response with subsequent stimuli (D). Other enhancers either had no effect (E) or slightly increased the probability of a response from the late (7-I0) stimuli (F). 


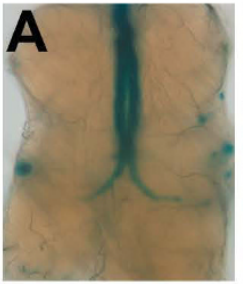

A307

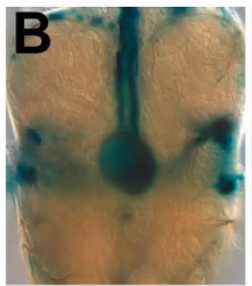

$A 307>\mathrm{GI}^{\mathrm{DN}}$

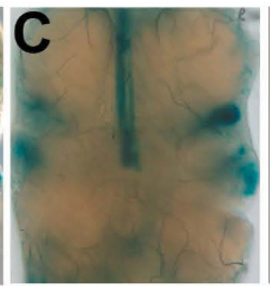

$A 307>\mathrm{Gl}^{\mathrm{DN}}$

$+a P K C^{06403 /+}$

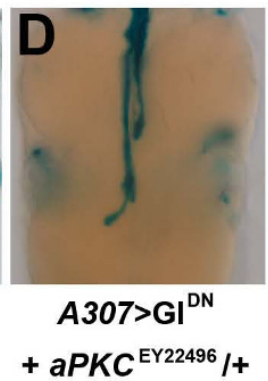

aPKC $C^{\mathrm{EY} 22496} /+$
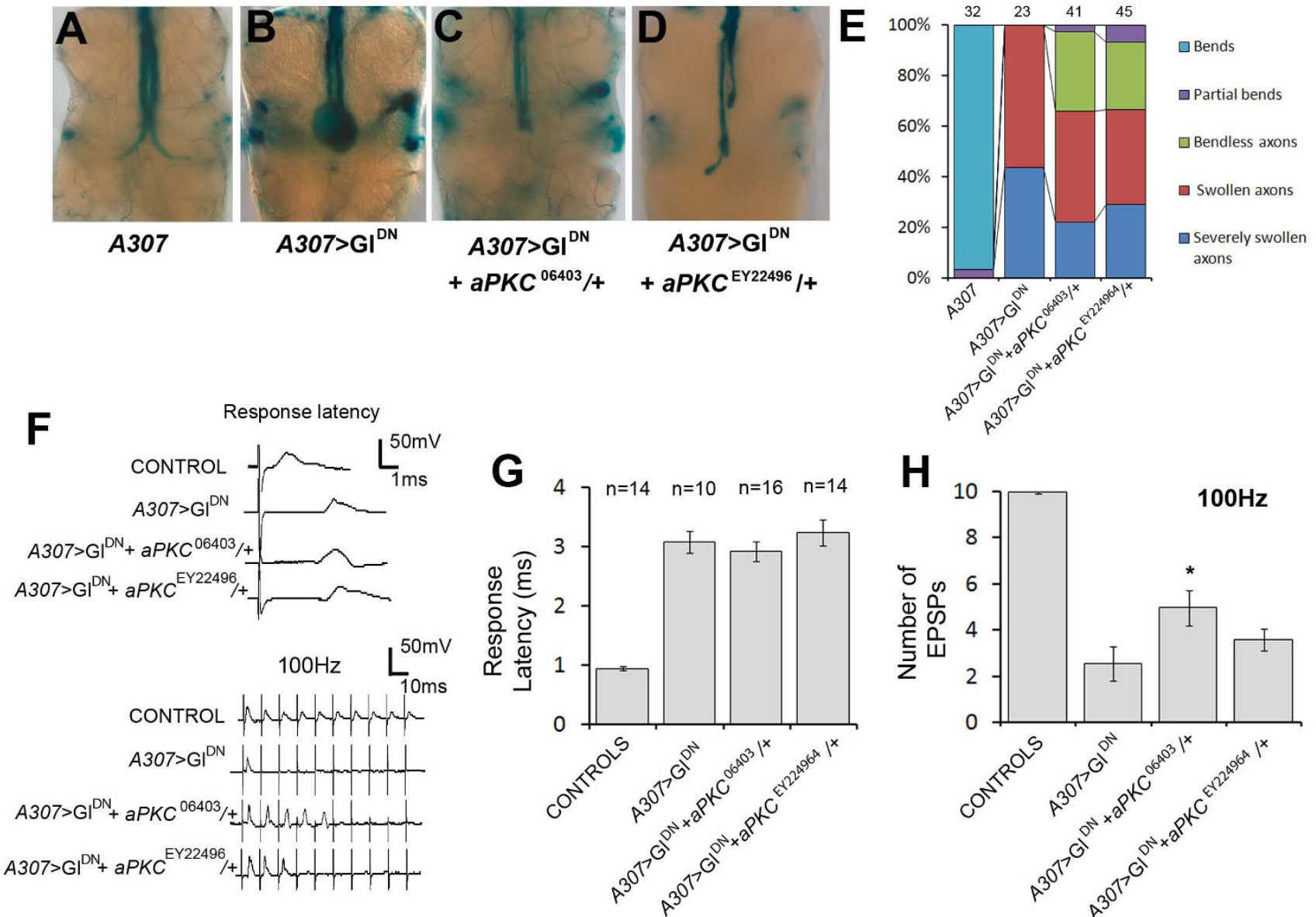

Figure 6

Mutations in aPKC suppress the $A 307>$ GIDN phenotype. (A-D) Dissected adult nervous systems stained for LacZ expression. Addition of a copy of either aPKC allele to the A307>GIDN background (C\&D) suppresses the swollen and bendless axon tips seen in $A 307>\left.G\right|^{D N}$ preparations (B). (E) Quantification of the morphological phenotypes revealing the extent of suppression. Numbers of GFs scored are given above bars. Severely swollen axons were those that were $>3$ times the normal diameter. (F) Traces from individual flies showing the response latency upon a single stimulus and following to 10 stimuli at 100 $\mathrm{Hz}$ in controls, A307>GIDN flies, and those also carrying the $a P K C$ alleles tested. (G\&H) Histograms showing the average response latencies and following at $100 \mathrm{~Hz}$ for the same genotypes. ${ }^{\mathrm{P}} \mathrm{P}<0.05$ in unpaired Student's $\mathrm{t}$-test. Controls were a mixture of UAS-GIDN, aPKC06403/+ and UAS-GIDN, aPKCEY224964/+ flies that did not carry A307.

weaker $G l^{1} /+$ eye phenotype were not obvious, however, SEM and sectioning was performed to show interactions with two of the mutations (EG37 \& SG13).

We used two different disruptions of Glued function, one strong and the other weaker, to assay successfully the effects of both enhancer and suppressor mutations in the GFS using both morphological and electrophysiological criteria. The severe disruptions of GF morphology and synaptic function enabled the effects of suppressor mutations to be clearly observed. This was less reliable when assaying the effects of mutations isolated as enhancers as either no increase of the already severe phenotype was seen or the interaction was lethal. For the enhancers, therefore, we relied on generating double heterozygotes with $G l^{1} /+$. As was the case in the eye, interactions were less pronounced and only two enhancers, EG37/+ and EG162/+ showed enhancement of the Gl1/+ electrophysiological phenotype. Indeed, the subtlety of some interactions with $G l^{1} /+$ may have resulted in our analyses being unable to detect some positive interacting loci in the GFS that altered the eye phenotype caused by GlDN.

We have generated some EMS alleles, two of which we have mapped to known genetic loci (see below) and four of which we have mapped to discrete chromosomal locations (Table 2). However, these four complement all the available lethal alleles in these regions indicating that our 
Table 2: Location of mutations isolated in the screen

\begin{tabular}{cc}
\hline Mutation & Chromosomal location or gene \\
\hline SGI3 & $60 \mathrm{~F} 5$ \\
SG46 & $22 \mathrm{~F}$ \\
SG58 & $a P K C$ \\
EG28 & $84 \mathrm{F7}-12$ \\
EG37 & $\mathrm{Su}(\mathrm{H})$ \\
EGI62 & $46 \mathrm{D}-\mathrm{E}$
\end{tabular}

mutations lie in loci for which there are few or no lethal alleles available. Identification of the location of these new alleles will require either new rounds of mutagenesis, such as via P-element excision in the mapped regions, finer mapping using SNPs [36-38] or custom made deficiencies using stocks from the DrosDel project [39]. Completion of the BDGP Gene Disruption Project may also enable mapping of the lesions [40] along with more recent approaches using other transposable elements that may disrupt genes refractory to P-element disruption [4143]. Interestingly, we appear not to have isolated any mutations in genes that encode known components of the retrograde motor complex including any further alleles of Glued. During some of the early genetic analysis of the Glued locus, dominant second-site suppressors of the $\mathrm{Gl}^{1}$ eye phenotype were isolated and reported [27]. Of these, two were mapped to the $\mathrm{X}$ chromosome ( $\mathrm{Su}[\mathrm{Gl}] 27 \& S u$ [Gl]57,[27]) and the others, $S u(G l) 77 \& S u(G l) 102$ are alleles of Dynein heavy chain 64C [44,45]. From the map positions of our mutations, we have not re-isolated similar alleles. Because our primary screen involved making only the eye mutant for Glued, we could potentially isolate mutations that are lethal in combination with $\mathrm{Gl}^{1}$ and would, therefore, not have been isolated Harte and Kankel's screen. However, none of the enhancers tested were lethal with $G l^{1}$.

We have successfully isolated two new alleles of known genes, $S u(H)$ and $a P K C$. Of the two, we have shown that alleles of $a P K C$ genetically interact with Glued in the GFS and suppress the abnormalities in GF-TTMn synapse formation seen when the retrograde motor complex is compromised by GlDN. These abnormalities are: a lack of the presynaptic "bends"; a branching event that takes place after the two neurons have met [31]; swollen axon tips and a weak or absent functional synapse [20]. aPKC is part of a protein complex, with PAR-3 (Bazooka in Drosophila) and PAR6 that regulates cell polarity in a number of different tissues/cells of Drosophila and vertebrates including neurons [46-48]. So what is the role of $a P K C$ in the GF neuron? In vertebrate neurons aPKC is needed for neurite outgrowth [49-51]. In contrast, aPKC in flies is an essential part of the machinery that polarizes dividing neuroblasts [52] but is not needed postmitotically for outgrowth
[53]. Our data also indicate that aPKC is not needed for neurite extension since the introduction of $a P K C$ mutations into our sensitized background has no effect on GF outgrowth. $a P K C$ is involved in memory formation in Drosophila [54] and at the developing larval NMJ it regulates microtubules (MTs) both pre- and postsynaptically during synapse formation [55]. Indeed MTs are one of the major targets of the PAR-3/PAR-6/aPKC complex in several contexts [56-58]. aPKC regulates MT orientation in fibroblasts $[59,60]$ and MT organization in the early embryo [61]. At the NMJ it controls MT stability with a reduction in aPKC activity causing a decreased association of MTs with the microtubule associated protein Futsch and MT fragmentation [55]. Dynein-dynactin is known to be involved in MT organization during growth cone remodeling as well as polarizing MTs in axons $[62,63]$. Our data indicate that dynein-dynactin and aPKC are acting antagonistically during formation of the GF presynaptic structure and suggest that both are needed to control microtubule organization and dynamics in synapse formation but have opposing roles. One simple explanation is that one of the roles of dynein-dynactin in the GF is to alter MT dynamics at the tip of the axon, when it has reached its post-synaptic target, so that they are more mobile enabling the presynaptic bend to be formed. aPKC regulates the stability of MTs thereby confining axon branching to a single bend. Blocking dynein-dynactin function prevents the MT re-organization needed for formation of the bends and this is ameliorated when aPKC function is reduced.

\section{Conclusion}

We have used a novel approach to screen for genes involved in central synapse formation by performing a primary screen, using a sensitized background, on the adult eye and then a secondary screen, on the isolated mutations, for synaptic phenotypes. This study shows that forward genetic screens are powerful tools for identifying genes with roles in CNS development.

\section{Methods \\ Drosophila strains}

The A307 [GAL4] enhancer-trap line and the line containing the UAS-GIDN transgene on chromosome 2 (UAS$\mathrm{Gl}^{\Delta 96 \mathrm{~B}}$ ) have been described previously $[20,33,64]$, as has the eye specific GMR-GAL4 line [65]. Stocks containing balancers, deficiency stocks, lethal alleles, aPKC ${ }^{06403 \text {, }}$ $a P K C^{\mathrm{EY} 22496}$ and $S u(H)^{1}$ flies were obtained from the Bloomington Drosophila Stock Center, Indiana, USA.

\section{GIDN over-expression screen}

$w$; UAS-GlDN males, isogenized on the second and third chromosomes, were mutagenized by feeding overnight with a $0.25 \mathrm{mM}$ solution of EMS in $1 \%$ sucrose. This dose was to generate, on average, only one lethal mutation per 
genome to facilitate downstream analysis. The mutagenized males were then mated to $w$; GMR-GAL4; TM6B/ $M K R S$ virgin females and the eyes of the progeny were scored for an enhancement or suppression of the GlDN eye phenotype. We isolated 324 adults with altered eye morphology; 215 potential enhancers and 109 potential suppressors. Mutations were recovered by mating flies with altered eye phenotypes individually to $w ; \mathrm{CyO} / \mathrm{Sco}$; $M K R S / T M 6 B$ flies. Sibling crosses were then performed to obtain lines with recessive lethal, or recessive viable, mutations on chromosomes 2 or 3 balanced over $\mathrm{CyO}$, $M K R S$ or $T M G B$. One hundred and sixteen lines were recovered with either a recessive lethal on chromosome 2 , 3 , or both. We did not obtain any recessive mutations that gave a homozygous visible phenotype. The 116 lines were crossed back to $w$; GMR-GAL4; TM6B/MKRS flies to confirm that the recovered mutations altered the GMR-GAL4/ UAS-GIDN eye phenotype. This resulted in 26 lines containing mutations that enhanced the phenotype and 15 lines that suppressed the phenotype.

\section{Scanning electron microscopy (SEM) and Retinal sectioning}

Whole flies were fixed in 2\% glutaraldehyde and dehydrated in acetone. Samples were dried in a Polatron E3000 critical point dryer, mounted onto stubs, and coated with gold. Micrographs were taken on a Hitachi S-430 electron microscope. For retinal sections, head cases were dissected from whole flies and fixed in 2\% glutaraldehyde, $2 \%$ paraformaldehyde in $0.1 \mathrm{M}$ PBS $(0.1 \mathrm{M} \mathrm{NaCl}, 0.1 \mathrm{M}$ $\mathrm{Na}_{2} \mathrm{HPO}_{4} / \mathrm{NaH}_{2} \mathrm{PO}_{4}$ [pH 6.8]) overnight at $4^{\circ} \mathrm{C}$. Following dehydration in an acetone series, the head cases were embedded in Durcupan resin and $2.5 \mu \mathrm{m}$ sections cut with a Lieca: Jung RM2065 microtome. Sections were stained with Toluidine blue and photographed on a Leica DMR microscope using a Leica DC500 digital camera.

\section{CNS Histochemistry}

The central nervous systems were dissected from adult flies in $0.1 \mathrm{M}$ PBS with $0.05 \%$ Triton X-100. For X-Gal staining they were then fixed in $1 \%$ gluteraldehyde for 5 min. Preparations were washed in PBT (0.1 M PBS, $0.1 \%$ Triton X-100) and pre-warmed in $2 \mathrm{mls}$ of X-Gal staining solution $\left(3 \mathrm{mM} \mathrm{K}_{4}\left[\mathrm{Fe}(\mathrm{CN})_{6}\right], 3 \mathrm{mM} \mathrm{K}_{3}\left[\mathrm{Fe}(\mathrm{CN})_{6}\right], 1 \mathrm{mM}\right.$ $\mathrm{MgCl}_{2}, 150 \mathrm{mM} \mathrm{NaCl}, 10 \mathrm{mM} \mathrm{Na}{ }_{2} \mathrm{HPO}_{4} / \mathrm{NaH}_{2} \mathrm{PO}_{4}[\mathrm{pH}$ 7.2 ], $0.3 \%$ Triton $\mathrm{X}-100$ ) in a watch glass for $5 \mathrm{~min}$ at $37^{\circ} \mathrm{C}$. To this was added $1 \mathrm{ml}$ of staining solution, saturated with dissolved X-Gal (5-bromo-4-chloro-3-indolyl $\beta$-D-galactopyranoside) and pre-warmed to $37^{\circ} \mathrm{C}$, and the preparations were incubated for several hours until staining of the GFs was complete.

\section{Electrophysiology}

Recordings from the GFS of adult flies were made essentially as described in [20]; a method based on those described by [66] and [67]. Flies were cooled on ice until they were immobile and secured in wax, ventral side down, with the wings held outwards in the wax. The GFs were stimulated using a Grass S48 stimulator to deliver a $40 \mathrm{~V}$ pulse for $0.03 \mathrm{~ms}$ through tungsten electrodes pushed through the eyes and into the brain. A tungsten earth wire was placed into the abdomen. Glass microelectrodes (resistance 40-60 M $\Omega$ ), filled with $3 \mathrm{M} \mathrm{KCl}$, were driven through the cuticle into the TTM and DLM muscles to record responses. These were amplified using Getting 5A amplifiers (Getting Instruments, USA) and the data digitized using an analogue-digital Digidata 1320 and Axoscope 9.0 software (Axon Instruments, USA). A single pulse was delivered for response latency measurements and trains of 10 stimuli, either $4 \mathrm{~ms}(250 \mathrm{~Hz})$ or $10 \mathrm{~ms}$ $(100 \mathrm{~Hz})$ apart, were given with a $5 \mathrm{~s}$ interval between each train for following frequency recordings. We routinely recorded from both TTM and a DLM in each preparation to ensure that correct stimulation of the GF was achieved. However, only data from the TTM are presented in this report.

\section{Genetic mapping}

Mutations were mapped using the "classic"second chromosome "deficiency kit" (DK2) of 110 stocks from the Bloomington Drosophila Stock Center containing mostly deletions that were defined cytologically. Once deficiencies were identified that failed to complement the isolated lethal mutations, smaller deficiencies in the regions were tested for complementation including molecularly defined deficiencies recently made available from Exelixis and the DrosDel project $[39,68]$. Standard complementation tests were then performed with known lethal mutations in the chromosomal regions uncovered by the deficiencies. SG13 failed to complement $D f(2 R) k r 10$ but complemented $D f(2 R) g s b$ and $D f(2 R) k r 14$ indicating that SG13 lies at 60F5. SG46 failed to complement $D f(2 L) 79 b$ which covers $22 \mathrm{~A} 2$ to 22E1. Testing smaller deletions within this region revealed that SG46 failed to complement the molecularly defined deletions $D f(2 L)$ Exel17008 (22B8;22D1) and Df(2L)Exel17011 (22E1;22F3). However when crossed together the two deletions failed to complement each other and both fail to complement alleles of $D p p$ which lies at 22F1-2. Therefore they both delete at least the region 22E1 to $22 \mathrm{~F} 3$ and $D f(2 L)$ Exel17008 is incorrectly annotated. SG58 failed to complement $D f(2 R) K n S A 4$ and $D f(2 R) X T E-58$ indicating it resided between $51 \mathrm{D} 1$ and 51D6 on chromosome 2. Using the available lethal mutations we determined SG58 to be an allele of Drosophila atypical Protein Kinase $C(a P K C)$ as it failed to complement $a P K C^{06403}$ and $a P K C^{\mathrm{EY} 22496}$, both known null alleles [52]. EG28 failed to complement $\mathrm{Df}(3 R) p 13$ which extends from $84 \mathrm{~F} 1$ to $85 \mathrm{~A} 8$. Within this region EG28 failed to complement $D f(3 R)$ exel6417 but complemented $D f(3 R) d s \times 29$ making its location to be 
within 84F7-84F12. All available lethal mutations were found to be not allelic to EG28. EG37 failed to complement $D f(2 L) T E 35 B C-24$ and $D f(2 L) A 48$ and testing known lethal alleles uncovered by both these deficiencies revealed the mutation to be in the $S u(H)$ locus. EG37 is lethal when trans-heterozygous with the $S u(H)^{1}$ allele. EG162 failed to complement $D f(2 R) X 1$ and $D f(2 R) \operatorname{stan} 1$ indicating that is resides between $46 \mathrm{D}$ and $47 \mathrm{~A}$. However, it did complement $D f(2 R)$ stan 2 indicating that it lies at $46 \mathrm{D}-\mathrm{E}$. We have been unable to map this mutation further at present.

\section{Authors' contributions}

LM and LAJ carried out the genetic screen; LAJ performed the SEM and eye sectioning; LM and MJA did the electrophysiology, all authors performed CNS dissection and staining. MJA designed and coordinated the work and drafted the manuscript. All authors read and approved the final manuscript.

\section{Acknowledgements}

Thank you to Kevin Moffat and Nara Muraro for useful discussions and help with the retinal sections, thanks to Ray Newsam and lan Brown for help with the SEM. Thank you to Dr Dan Mulvihill for critical reading of the manuscript. This work was supported by a BBSRC studentship to LAJ and a Wellcome Trust Project Grant (0697/0/Z/02/Z) to MJA.

\section{References}

I. Davis GW: The making of a synapse: Target-derived signals and presynaptic differentiation. Neuron 2000, 26(3):55I-554

2. Jin Y, Garner CC: Molecular mechanisms of presynaptic differentiation. Annu Rev Cell Dev Biol 2008, 24:237-262.

3. Allen MJ, Godenschwege TA, Tanouye MA, Phelan P: Making an escape: development and function of the Drosophila giant fibre system. Semin Cell Dev Biol 2006, I 7(I):3I-4I.

4. Godenschwege TA, Hu H, Shan-Crofts X, Goodman CS, Murphey RK: Bi-directional signaling by Semaphorin Ia during central synapse formation in Drosophila. Nat Neurosci 2002, 5(I 2): | 294- | 30|

5. Godenschwege TA, Simpson JH, Shan X, Bashaw GJ, Goodman CS, Murphey RK: Ectopic expression in the giant fiber system of Drosophila reveals distinct roles for roundabout (Robo), Robo2, and Robo3 in dendritic guidance and synaptic connectivity. I Neurosci 2002, 22(8):3117-3I29.

6. Godenschwege TA, Kristiansen LV, Uthaman SB, Hortsch M, Murphey RK: A conserved role for Drosophila Neuroglian and human LI-CAM in central-synapse formation. Curr Biol 2006, I 6(I):12-23

7. Muralidhar MG, Thomas JB: The Drosophila Bendless Gene Encodes a Neural Protein Related to Ubiquitin-Conjugating Enzymes. Neuron 1993, I I(2):253-266.

8. Murphey RK, Froggett SJ, Caruccio P, Shan-Crofts X, Kitamoto T, Godenschwege TA: Targeted expression of shibire ts and semaphorin Ia reveals critical periods for synapse formation in the giant fiber of Drosophila. Development 2003, I 30( I 6):367|-3682.

9. Oh CE, McMahon R, Benzer S, Tanouye MA: Bendless, a Drosophila Gene Affecting Neuronal Connectivity, Encodes a Ubiquitin-Conjugating Enzyme Homolog. Journal Of Neuroscience 1994, I4(5 Pt 2):3166-3179.

10. Uthaman SB, Godenschwege TA, Murphey RK: A mechanism distinct from highwire for the Drosophila ubiquitin conjugase bendless in synaptic growth and maturation. J Neurosci 2008 , 28(34):8615-8623.

II. Allen MJ, Shan X, Murphey RK: A role for Drosophila Dracl in neurite outgrowth and synaptogenesis in the giant fiber system. Mol Cell Neurosci 2000, I 6(6):754-765.
12. Allen MJ, Drummond JA, Sweetman DJ, Moffat KG: Analysis of two P-element enhancer-trap insertion lines that show expression in the giant fibre neuron of Drosophila melanogaster. Genes Brain Behav 2007, 6(4):347-358.

13. Schroer TA: Dynactin. Annu Rev Cell Dev Biol 2004, 20:759-779.

14. Watermanstorer CM, Holzbaur ELF: The Product Of the Drosophila Gene, Glued, Is the Functional Homolog Of the PI50(Glued) Component Of the Vertebrate Dynactin Complex. Journal Of Biological Chemistry 1996, 27 I (2): I I53-I I 59.

15. Swaroop A, Paco-Larson ML, Garen A: Molecular genetics of a transposon-induced dominant mutation in the Drosophila locus Glued. Proc Natl Acad Sci USA 1985, 82(6): |75।-I755.

16. McGrail M, Gepner J, Silvanovich A, Ludmann S, Serr M, Hays TS: Regulation Of Cytoplasmic Dynein Function In-Vivo By the Drosophila Glued Complex. Journal Of Cell Biology 1995 , | 3 | (2):4 | |-425.

17. Fan SS, Ready DF: Glued participates in distinct microtubulebased activities in Drosophila eye development. Development I997, I 24(8): | 497-I507.

18. Murphey RK, Caruccio PC, Getzinger M, Westgate PJ, Phillis RW: Dynein-dynactin function and sensory axon growth during Drosophila metamorphosis: A role for retrograde motors. Dev Biol 1999, 209(I):86-97.

19. Reddy S, Jin P, Trimarchi J, Caruccio P, Phillis R, Murphey RK: Mutant molecular motors disrupt neural circuits in Drosophila. J Neurobiol 1997, 33(6):7II-723.

20. Allen MJ, Shan X, Caruccio P, Froggett SJ, Moffat KG, Murphey RK: Targeted expression of truncated glued disrupts giant fiber synapse formation in Drosophila. J Neurosci 1999, I9(2I):9374-9384.

21. Reuter JE, Nardine TM, Penton A, Billuart P, Scott EK, Usui T, Uemura T, Luo L: A mosaic genetic screen for genes necessary for Drosophila mushroom body neuronal morphogenesis. Development 2003, I30(6): | 203-1213.

22. Karim FD, Chang HC, Therrien M, Wassarman DA, Laverty T, Rubin GM: A screen for genes that function downstream of Ras I during Drosophila eye development. Genetics 1996 I 43(1):315-329.

23. Carrera P, Abrell S, Kerber B, Walldorf U, Preiss A, Hoch M, Jackle $\mathrm{H}$ : A modifier screen in the eye reveals control genes for Kruppel activity in the Drosophila embryo. Proc Natl Acad Sci USA 1998, 95( I 8): I0779-10784.

24. Mahoney MB, Parks AL, Ruddy DA, Tiong SY, Esengil H, Phan AC, Philandrinos P, Winter CG, Chatterjee R, Huppert K, et al.: Presenilinbased genetic screens in Drosophila melanogaster identify novel notch pathway modifiers. Genetics 2006, I 72(4):2309-2324.

25. Thomas BJ, Wassarman DA: A fly's eye view of biology. Trends Genet 1999, I5(5): 184-190.

26. Thaker HM, Kankel DR: Mosaic analysis gives an estimate of the extent of genomic involvement in the development of the visual system in Drosophila melanogaster. Genetics 1992 , I 3 | (4):883-894.

27. Harte PJ, Kankel DR: Genetic-Analysis Of Mutations At the Glued Locus and Interacting Loci In Drosophila-Melanogaster. Genetics 1982, I0I(3-4):477-50I.

28. Brand AH, Perrimon N: Targeted Gene-Expression As a Means Of Altering Cell Fates and Generating Dominant Phenotypes. Development 1993, I I 8(2):401-4I5.

29. Kramer JM, Staveley BE: GAL4 causes developmental defects and apoptosis when expressed in the developing eye of Drosophila melanogaster. Genet Mol Res 2003, 2(I):43-47.

30. Blagburn JM, Alexopoulos H, Davies JA, Bacon JP: Null mutation in shaking-B eliminates electrical, but not chemical, synapses in the Drosophila giant fiber system: a structural study. J Comp Neurol 1999, 404(4):449-458.

31. Jacobs K, Todman MG, Allen MJ, Davies JA, Bacon JP: Synaptogenesis in the giant-fibre system of Drosophila: interaction of the giant fibre and its major motorneuronal target. Development 2000, I 27(23):5203-52। 2.

32. Koto M, Tanouye MA, Ferrus A, Thomas JB, Wyman RJ: The Morphology Of the Cervical Giant Fiber Neuron Of Drosophila. Brain Research 198I, $221(2): 213-217$.

33. Phelan P, Nakagawa M, Wilkin MB, Moffat KG, O'Kane CJ, Davies JA, Bacon JP: Mutations in shaking-B prevent electrical synapse 
formation in the Drosophila giant fiber system. I Neurosci 1996, 16(3): II0I-III3.

34. Harte PJ, Kankel DR: Analysis of visual system development in Drosophila melanogaster: mutations at the Glued locus. Dev Biol 1983, 99(I):88-102.

35. Whited JL, Cassell A, Brouillette M, Garrity PA: Dynactin is required to maintain nuclear position within postmitotic Drosophila photoreceptor neurons. Development 2004, I 3 I ( 19):4677-4686

36. Hoskins RA, Phan AC, Naeemuddin M, Mapa FA, Ruddy DA, Ryan JJ, Young LM, Wells T, Kopczynski C, Ellis MC: Single nucleotide polymorphism markers for genetic mapping in Drosophila melanogaster. Genome Res 200I, I I(6): I I00-IIII3.

37. Martin SG, Dobi KC, St Johnston D: $\mathbf{A}$ rapid method to map mutations in Drosophila. Genome Biol 200I, 2(9): RESEARCH0036.

38. Chen D, Berger J, Fellner M, Suzuki T: FLYSNPdb: a high-density SNP database of Drosophila melanogaster. Nucleic acids research 2009:D567-570.

39. Ryder E, Blows F, Ashburner M, Bautista-Llacer R, Coulson D, Drummond J, Webster J, Gubb D, Gunton N, Johnson G, et al.: The DrosDel collection: a set of P-element insertions for generating custom chromosomal aberrations in Drosophila melanogaster. Genetics 2004, 167(2):797-8I3.

40. Bellen HJ, Levis RW, Liao G, He Y, Carlson JW, Tsang G, Evans-Holm M, Hiesinger PR, Schulze KL, Rubin GM, et al.: The BDGP gene disruption project: single transposon insertions associated with 40\% of Drosophila genes. Genetics 2004, 167(2):76I-78I.

4I. Hacker U, Nystedt S, Barmchi MP, Horn C, Wimmer EA: piggyBacbased insertional mutagenesis in the presence of stably integrated $\mathbf{P}$ elements in Drosophila. Proc Natl Acad Sci USA 2003, 100(13):7720-7725

42. Horn C, Offen N, Nystedt S, Hacker U, Wimmer EA: piggyBacbased insertional mutagenesis and enhancer detection as a tool for functional insect genomics. Genetics 2003, 163(2):647-661.

43. Schuldiner O, Berdnik D, Levy JM, Wu JS, Luginbuhl D, Gontang AC, Luo L: piggyBac-based mosaic screen identifies a postmitotic function for cohesin in regulating developmental axon pruning. Developmental cell 2008, I 4(2):227-238.

44. lyadurai SJ, Robinson JT, Ma L, He Y, Mische S, Li MG, Brown W, Guichard A, Bier E, Hays TS: Dynein and Star interact in EGFR signaling and ligand trafficking. Journal of cell science 2008, I 2 I (Pt 16):2643-2651.

45. McGrail M, Gepner J, Silvanovich A, Ludmann S, Serr M, Hays TS: Regulation of cytoplasmic dynein function in vivo by the Drosophila Glued complex. J Cell Biol I995, I3 I (2):4| I-425.

46. Macara IG: Parsing the polarity code. Nature reviews 2004, 5(3):220-231.

47. Wiggin GR, Fawcett JP, Pawson T: Polarity proteins in axon specification and synaptogenesis. Developmental cell 2005, 8(6):803-816

48. Wodarz A: Molecular control of cell polarity and asymmetric cell division in Drosophila neuroblasts. Current opinion in cell biology 2005, I7(5):475-48I.

49. Shi SH, Jan LY, Jan YN: Hippocampal neuronal polarity specified by spatially localized mPar3/mPar6 and PI 3-kinase activity. Cell 2003, I I 2(I):63-75.

50. Nishimura T, Kato K, Yamaguchi T, Fukata Y, Ohno S, Kaibuchi K: Role of the PAR-3-KIF3 complex in the establishment of neuronal polarity. Nature cell biology 2004, 6(4):328-334.

51. Schwamborn JC, Puschel AW: The sequential activity of the GTPases RapIB and Cdc42 determines neuronal polarity. Nat Neurosci 2004, 7(9):923-929.

52. Rolls MM, Albertson R, Shih HP, Lee CY, Doe CQ: Drosophila aPKC regulates cell polarity and cell proliferation in neuroblasts and epithelia. J Cell Biol 2003, I63(5):1089-1098.

53. Rolls MM, Doe CQ: Baz, Par-6 and aPKC are not required for axon or dendrite specification in Drosophila. Nat Neurosci 2004, 7( I 2): | 293-1295.

54. Drier EA, Tello MK, Cowan M, Wu P, Blace N, Sacktor TC, Yin JC: Memory enhancement and formation by atypical PKM activity in Drosophila melanogaster. Nat Neurosci 2002, 5(4):316-324.

55. Ruiz-Canada C, Ashley J, Moeckel-Cole S, Drier E, Yin J, Budnik V: New synaptic bouton formation is disrupted by misregula- tion of microtubule stability in aPKC mutants. Neuron 2004 , 42(4):567-580.

56. Etienne-Manneville S, Hall A: Cell polarity: Par6, aPKC and cytoskeletal crosstalk. Current opinion in cell biology 2003, I5(I):67-72.

57. Suzuki A, Ohno S: The PAR-aPKC system: lessons in polarity. Journal of cell science 2006, I I 9(Pt 6):979-987.

58. Wodarz A: Establishing cell polarity in development. Nature cell biology 2002, 4(2):E39-44.

59. Cau J, Hall A: Cdc42 controls the polarity of the actin and microtubule cytoskeletons through two distinct signal transduction pathways. Journal of cell science 2005 , I / $\mathbf{8}(\mathbf{P t}$ I 2):2579-2587.

60. Etienne-Manneville S, Hall A: Cdc42 regulates GSK-3beta and adenomatous polyposis coli to control cell polarity. Nature 2003, 42 I (6924):753-756.

61. Harris T], Peifer M: aPKC controls microtubule organization to balance adherens junction symmetry and planar polarity during development. Developmental cell 2007, I 2(5):727-738.

62. Grabham PW, Seale GE, Bennecib M, Goldberg DJ, Vallee RB: Cytoplasmic dynein and LISI are required for microtubule advance during growth cone remodeling and fast axonal outgrowth. I Neurosci 2007, 27(2 I):5823-5834.

63. Zheng Y, Wildonger J, Ye B, Zhang Y, Kita A, Younger SH, Zimmerman $S$, Jan $L Y$, Jan $Y N$ : Dynein is required for polarized dendritic transport and uniform microtubule orientation in axons. Nature cell biology 2008, I0(10): I 172-1 I 80.

64. Allen MJ, Drummond JA, Moffat KG: Development of the giant fiber neuron of Drosophila melanogaster. J Comp Neurol 1998, 397(4):519-531.

65. Freeman M: Reiterative use of the EGF receptor triggers differentiation of all cell types in the Drosophila eye. Cell 1996, 87(4):65I-660.

66. Tanouye MA, Wyman RJ: Motor outputs of giant nerve fiber in Drosophila. J Neurophysiol I 980, 44(2):405-42I.

67. Gorczyca M, Hall JC: Identification of a cholinergic synapse in the giant fiber pathway of Drosophila using conditional mutations of acetylcholine synthesis. J Neurogenet 1984, I(4):289-3 I3.

68. Parks AL, Cook KR, Belvin M, Dompe NA, Fawcett R, Huppert K, Tan LR, Winter CG, Bogart KP, Deal JE, et al.: Systematic generation of high-resolution deletion coverage of the Drosophila melanogaster genome. Nat Genet 2004, 36(3):288-292.

\section{Publish with BioMed Central and every scientist can read your work free of charge}

"BioMed Central will be the most significant development for disseminating the results of biomedical research in our lifetime. "

Sir Paul Nurse, Cancer Research UK

Your research papers will be:

- available free of charge to the entire biomedical community

- peer reviewed and published immediately upon acceptance

- cited in PubMed and archived on PubMed Central

- yours - you keep the copyright
BioMedcentral 